\title{
BMJ Global Health Are community health workers effective in retaining women in the maternity care continuum? Evidence from India
}

\author{
Smisha Agarwal, ${ }^{\oplus 1,2,3}$ Sian Curtis, ${ }^{\oplus 3,4}$ Gusavo Angeles, ${ }^{3,4}$ Ilene Speizer, ${ }^{3,4}$ \\ Kavita Singh, ${ }^{3,4}$ James Thomas ${ }^{4,5}$
}

To cite: Agarwal S, Curtis S, Angeles G, et al. Are community health workers effective in retaining women in the maternity care continuum? Evidence from India. BMJ Global Health 2019:4:e001557. doi:10.1136/ bmjgh-2019-001557

Handling editor Stephanie M Topp

Received 11 March 2019

Revised 28 May 2019

Accepted 1 June 2019

Check for updates

(c) Author(s) (or their employer(s)) 2019. Re-use permitted under CC BY-NC. No commercial re-use. See rights and permissions. Published by BMJ

${ }^{1}$ Department of International Health, Johns Hopkins University Bloomberg School of Public Health, Baltimore, Maryland USA

${ }^{2}$ University of North Carolina at Chapel Hill Carolina Population Center, Chapel Hill, North Carolina, USA

${ }^{3}$ Department of Maternal and Child Health, University of North Carolina at Chapel Hill Gillings School of Global Public Health, Chapel Hill, North Carolina, USA ${ }^{4}$ MEASURE Evaluation, University of North Carolina at Chapel Hill Carolina Population Center, Chapel Hill, North Carolina, USA ${ }^{5}$ Department of Epidemiology, University of North Carolina at Chapel Hill Gillings School of Global Public Health, Chapel Hill, North Carolina, USA

Correspondence to Dr Smisha Agarwal; smishaa@gmail.com

\section{ABSTRACT}

Objectives Despite the recognised importance of adopting a continuum of care perspective in addressing the care of mothers and newborns, evidence on specific interventions to enhance engagement of women along the maternity care continuum has been limited. We use the example of the Accredited Social Health Activist (ASHA) programme in India, to understand the role of community health workers in retaining women in the maternity care continuum. Methods Using the Indian Human Development Survey data from 2011 to 2012, we assess the association between individual and cluster-level exposure to ASHA and four key components along the continuum of care-at least one antenatal care (ANC) visit, four or more ANC visits, presence of a skilled birth attendance (SBA) at the time of birth and postnatal care for the mother or child within 48 hours of birth, for 13705 women with a live birth since 2005. To understand which of these services experience maximum dropout along the continuum, we use a linear probability model to calculate the weighted percentages of using each service. We assess the association between exposure to ASHA and number of services utilised using a multinomial logistic regression model adjusted for a range of confounding variables and survey weights.

Results Our study indicates that exposure to the ASHA is associated with an increased probability of women receiving at least one ANC and SBA. In terms of numbers of services, exposure to ASHA accounts for a $12 \%(95 \%$ Cl: 9.1 to 15.1 ) increase in women receiving at least some of the services, and an $8.8 \%(95 \% \mathrm{Cl}:-10.2$ to $-7.4)$ decrease in women receiving no services. However, exposure to ASHA does not increase the likelihood of women utilising all the services along the continuum. Conclusions While ASHA is effective in supporting women to initiate and continue care along the continuum, it does not significantly affect the completion of all services along the continuum.

\section{BACKGROUND}

The continuum of care is an essential principle of programme targeting improvements in maternal, newborn and child health. The continuum of care is defined along two dimensions - throughout the life cycle

\section{Key questions}

What is already known?

- Integrated services that address the entire continuum from the antepartum to the postnatal period are important for gains in maternal and child survival.

- One approach that has often been used to enhance engagement along the continuum involves training community health workers (CHWs) to engage with pregnant and postpartum women.

- However, this approach has not been empirically tested.

What are the new findings?

- This study evaluates the role of the Accredited Social Health Activists programme (ASHA) in India- one of the largest CHW programme - on supporting women to initiate care during the antenatal period and on ensuring retention in care through the postnata period.

- The study finds that the ASHA is effective in supporting initiation and continuation of engagement with services along the maternity continuum.

- However, it is not significantly associated with completion of all services.

\section{What do the new findings imply?}

- Our study suggests that the presence of an ASHA in a community encourages positive healthcare-seeking behaviours along the continuum of maternity care.

- While the presence of an ASHA can encourage health-seeking behaviour, to ensure continuity of care, efforts must also be directed at systemic interventions to ensure availability and quality of facility-level services.

including adolescence, pregnancy, childbirth, postnatal period and childhood, and across the levels of care from the household to the health facility. ${ }^{1}$ The continuity of care lens recognises the intergenerational gains for the mother and child achieved from intervening earlier during the adolescent and antepartum periods.

For most women in low-income countries, the first point of entry into the maternity care 
continuum is typically during pregnancy. Timely and appropriate antenatal care (ANC) affects not just the health of the mother but also the newborn..$^{2-5}$ Delayed initiation of ANC or fewer than four ANC visits is associated with preterm birth or low birth weight. ${ }^{67}$ There is a consistent link between ANC and skilled birth attendance (SBA) / facility-based delivery ${ }^{489}$; SBA is at least six times more likely among women who have had at least one ANC visit compared with women with no ANC. ${ }^{2}$ Globally, studies have shown a strong relationship between more than four ANC visits and delivering in a health facility. Similarly, a strong correlation exists between ANC and postnatal care (PNC), and SBA and PNC. Women who have four or more ANC visits or deliver with a skilled birth attendant are more likely to receive PNC. ${ }^{5}$ Timely and appropriate PNC also has significant benefits for both the mother and the child. ${ }^{10} 11$

More recently, studies have specifically adopted a continuum of care lens to identify demographic factors associated with the engagement of women along the maternity continuum. ${ }^{8912}$ Though the studies have slight variations in the components comprising the continuum, including family planning, one or more ANC visits, four ANC visits, SBA, facility births and PNC measured at 24 hours, 48 hours, 2 weeks and 6 weeks, common themes have emerged. Women who are wealthier, more educated and have greater autonomy are more likely to receive a greater number of health services. ${ }^{89} 12$ Less than one-quarter of women in the three South Asian countries and $15 \%$ in six sub-Saharan African countries receive all the elements along the continuum. ${ }^{9}$ All three studies emphasised the need for increased attention and research to understand factors that may improve retention along the continuum of maternity care.

One approach that has often been used to enhance engagement along the continuum involves training community health workers (CHWs) to engage with pregnant and postpartum women. ${ }^{13-15}$ CHWs can undertake a variety of tasks at the community level, including providing maternity stage-appropriate health education, connecting women to healthcare facilities and providing home-based ANC and PNC. ${ }^{13}{ }^{16}$ Globally, there has been a renewed interest in investing in CHWs programme for their potential impact on strengthening primary healthcare linkages for women and children. Several South Asian and African countries, including India, Bangladesh, Ethiopia, Kenya, Uganda, Ghana and South Africa, are investing in training and deploying cadres of CHWs to focus on maternal and child health. ${ }^{1317} 18$ Given their scale and the investments, it is important to assess the evidence on the effectiveness of these programme.

Our study evaluates the role of CHWs in retaining women in the maternity care continuum, using the example of the Accredited Social Health Activist (ASHA) programme in India. The ASHA programme in India is the largest CHW programme, with nearly one million ASHA's operating nationally. The ASHA plays a critical role at each point along the continuum-counsel pregnant women on birth preparedness, safe delivery; accompany mothers and their families to the health facility; and provide care and referrals for newborn and childhood ailments. ${ }^{19}$ We assess the association between exposure to the ASHA programme and four key components along the continuum of care-at least one ANC visit, four or more ANC visits, presence of a skilled attendant at the time of birth and PNC for the mother or child within 48 hours of birth, and explore how exposure to ASHA may influence the number of services utilised along the continuum.

\section{METHODS}

Data for this study come from the nationally representative Indian Human Development Survey (IHDS), funded by the National Institutes of Child Health and Human Development and produced by the National Council of Applied Economic Research, New Delhi and the University of Maryland. The study uses IHDS-II data from interviews with ever-married women aged 15-49 years, regarding health, education, fertility and family planning, collected in 2011-2012. ${ }^{20}$ IHDS-II comprises of data from 42152 households, across 33 states and union territories, 384 districts, 1420 villages and 1042 urban blocks. The rural sample was drawn using stratified random sampling, and the urban sample was a stratified sample of towns and cities within states, selected using probability proportional to the population size. Our study focused on data from 13705 women with a live birth since $2005 .^{20}$

\section{Patient and public involvement statement}

There was public involvement in the primary study. However, we used publicly available secondary data for the analysis.

\section{Study Measures}

The key outcome measures for this analysis were receipt of at least one ANC visit (ANC-1), receipt of four or more ANC visits (ANC-4), having a skilled attendant at birth (SBA) and receipt of PNC either for the mother or the child within 48 hours after birth (PNC). Medical doctors, nurses, and auxiliary skilled birth attendants are considered as skilled birth attendants. ${ }^{21}$

A woman was considered exposed to an ASHA if she responded that an ASHA assisted her in response to at least one of the following questions: 'Where did you get a pregnancy card made?'; 'Did you get help from anyone for making a pregnancy card/registration?'; 'Who visited you when you were pregnant?'; 'Who facilitated or motivated you to go to a health facility for delivery?' and 'Who arranged the transportation to take you to the health facility for delivery?' In addition to individual reports of exposure to ASHA, we sought to understand how community-level exposure to ASHA may affect individual's health-seeking behaviours. The mean cluster exposure value was calculated as the number of women who reported exposure to an ASHA in a cluster during the last birth divided by the total number of eligible women 
who had a birth in the 6 years preceding the survey in the cluster. This measure value ranges from 0 to 1 and can be interpreted as the 'intensity' of cluster-level exposure. This intensity of exposure was further categorised as 'no exposure', medium exposure (exposure values $>0$ to $<0.50$ ) and high exposure (exposure values $\geq 0.50$ to $\leq 1$ ).

\section{Analysis}

Several descriptive approaches were used to assess the association between the utilisation of individual services comprising the continuum and exposure to ASHA, and to identify the elements of the continuum that are most frequently missed. First, we used a matrix to assess which combination of services is frequently observed in the sample population. In the matrix, we categorise each service individually, and sum the number of services as 'none', 'some' (any one, two or three services) and 'all' services. Utilisation of 'some' services signifies service initiation and continuation along the continuum, and utilisation of 'all' services signifies completion of the continuum of services. We calculate the unadjusted weighted percentages of each of the individual services comprising the continuum by individual ASHA exposure, and by cluster-level ASHA intensity. Finally, we use a linear probability model to generate probabilities of utilising maternity services by individual and cluster-level ASHA exposure intensity, adjusted for confounding variables. Enrollment and retention within the continuum is not a sequential process. Some women may dropout of the continuum in the earlier stages (eg, at ANC-4), and may enter the continuum at a later stage (eg, for $\mathrm{SBA}$ ). These descriptive analyses help us assess where significant dropouts from services occurred, the effect of increasing ASHA intensity on the outcomes, and the change in the relationship between ASHA exposure and the outcomes when women's demographic characteristics are accounted for.

To understand how exposure to ASHA influences the utilisation of services across the continuum, we examined the relationship between exposure to ASHA and receipt of maternity services in two ways: (1) We modelled the outcomes categorised as 'none', 'some' and 'all' with the two exposure specifications of individual ASHA exposure, and cluster-level ASHA exposure intensity using a multinomial logistic regression model. (2) To look more closely at the association between exposure to ASHA and number of services, we fitted a multinomial logistic regression model with the number of services categorised as $0,1,2,3$ and 4 as the outcome with the two ASHA exposure specifications. Predicted probabilities, marginal effects and CIs were calculated across each level of the outcome using the margins command in Stata. Margins uses the delta method to estimate the standard errors of the marginal effect. ${ }^{22}$ All analyses, conducted using Stata V.14.1 (Stata Corp LP, College Station, TX, USA), are restricted to clusters with two or more women and accounts for survey weights.
We controlled for a number of potentially confounding variables. These included socio-demographic characteristics of the women, including maternal age (15-19, 20-24, 25-29, 30-34, 35-39, 40-49 years), maternal education (1-5 years, secondary $6-11$ years, 12 years or more of education), maternal caste (upper/forward caste, scheduled caste (SC), scheduled tribe (ST), other backward caste $(\mathrm{OBC})$ ), religion (Hindu, Muslim, other

Table 1 Demographic characteristics of sample population of women

\begin{tabular}{|cc}
\hline Characteristic & $\mathbf{N}=\mathbf{1 3} \mathbf{7 0 5}(\%)$ \\
\hline $\begin{array}{c}\text { Maternal age (years) } \\
15-19\end{array}$ & $297(2.17)$ \\
$20-24$ & $3133(22.86)$ \\
$25-29$ & $4986(36.38)$ \\
$30-34$ & $3151(22.99)$ \\
$35-39$ & $1434(10.46)$ \\
$>=40$ & $703(5.13)$ \\
\hline
\end{tabular}

\section{Education}

$\begin{array}{lc}\text { No education } & 4590(33.49) \\ \text { 1-5 years } & 2009(14.66) \\ 6-11 \text { years } & 5022(36.64) \\ 12 \text { years or more } & 2076(15.15) \\ \text { Missing } & 8(0.06) \\ \text { Caste } & \\ \text { Forward caste } & 3459(25.24) \\ \text { Other backward castes } & 5858(42.74) \\ \text { Scheduled castes } & 3180(23.20) \\ \text { Scheduled tribes } & 1181(8.62) \\ \text { Missing } & 27(0.20)\end{array}$

Religion

Hindu 11011 (80.34)

Muslim

$2094(15.28)$

Others religions

$600(4.38)$

\section{States}

\begin{tabular}{lc}
\hline Other states & $5930(43.27)$ \\
\hline High-focus states & $7260(52.97)$ \\
\hline Northeast states & $515(3.76)$ \\
Urban/rural & \\
Rural & $9757(71.19)$ \\
Urban & $3948(28.81)$ \\
Wealth quintile & \\
\hline Lowest & $3747(27.34)$ \\
\hline Lower & $2900(21.16)$ \\
Middle & $2524(18.42)$ \\
Richer & $2375(17.33)$ \\
Richest & $2159(15.57)$ \\
Parity (mean (SD)) & $2.589(1.68)$ \\
\hline
\end{tabular}


Table 2 Weighted percentages of women who received each combination of services along the continuum of care

\begin{tabular}{|c|c|c|c|c|c|c|c|c|}
\hline $\begin{array}{l}\text { No. of } \\
\text { services }\end{array}$ & $\begin{array}{l}\text { Number of } \\
\text { services }\end{array}$ & ANC-1 & ANC-4 & SBA & PNC & Weighted \% & $\begin{array}{l}\text { Weighted } \\
\% \text { (by no. of } \\
\text { services) }\end{array}$ & $\begin{array}{l}N=13 \\
705\end{array}$ \\
\hline 0 & None & & & & & 8.56 & 8.56 & 1173 \\
\hline \multirow[t]{3}{*}{1} & Some & $X$ & & & & 8.11 & 12.77 & 1111 \\
\hline & & & & $x$ & & 3.53 & & 484 \\
\hline & & & & & $x$ & 1.13 & & 155 \\
\hline \multirow[t]{4}{*}{2} & & $x$ & $x$ & & & 3.02 & 25.28 & 414 \\
\hline & & $x$ & & $x$ & & 16.43 & & 2252 \\
\hline & & $x$ & & & $x$ & 2.7 & & 370 \\
\hline & & & & $x$ & $x$ & 3.13 & & 429 \\
\hline \multirow[t]{3}{*}{3} & & $x$ & $x$ & $x$ & & 18.59 & 33.33 & 2548 \\
\hline & & $x$ & & $x$ & $x$ & 13.7 & & 1878 \\
\hline & & $x$ & $x$ & & $x$ & 1.04 & & 143 \\
\hline 4 & All & $X$ & 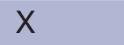 & $X$ & 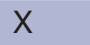 & 20.06 & 20.06 & 2749 \\
\hline
\end{tabular}

ANC, antenatal care; PNC, postnatal care.

religions), parity, and household wealth index. OBC, SC and ST are official Government of India caste classifications for groups of historically disadvantaged people. We used polychoric principal components analysis to estimate household wealth quintiles with information about household asset ownership and household characteristics from the IHDS household surveys. ${ }^{23}$

The University of North Carolina, Chapel Hill Institutional Review Board determined that this study meets the exempt criteria as it does not constitute human subjects research.

\section{RESULTS}

\section{Trends in the utilisation of maternity services along the continuum of care}

Over $60 \%$ of the women were under 30 years of age (table 1). One-third of the women had no formal education. Over $50 \%$ of the sample is from high-focus states, $43 \%$ from other states and $4 \%$ from northeastern states. In total, $71 \%$ of the sample is rural (table 1) and $25 \%$ of the women in the sample reported exposure to ASHA. Measured as cluster-level intensity, $41 \%$ women lived in clusters where no ASHA services were reported, 36\% lived in clusters where a 'medium' intensity of ASHA services was reported and $23 \%$ lived in clusters where 'high' ASHA intensity was reported.

Most women $(71.38 \%)$ utilised between one and three services along the maternity continuum, $8.5 \%$ utilised no services and $20 \%$ utilised all the four services (table 2). For women receiving one to three services, the most frequently observed combination was ANC-1, ANC-4 and facility birth. In all, $14 \%$ of the women received only one ANC and then went on to have a facility birth and PNC; and $16 \%$ of women received only one ANC and then went on to have a facility birth. Less than $5 \%$ of the women, who did not have a facility birth, went on to receive PNC.
Cascade of maternity services received by ASHA exposure status Women who report ASHA services had significantly higher reported use of ANC-1 and SBA, after accounting for women's demographic characteristics (figure 1). ANC-4 and PNC were the two services along the continuum that were most frequently missed. We also explored the relationship between cluster-level ASHA exposure intensity and outcomes (figure 2). We observe no significant differences in the use of services across the different levels of exposure intensity, after accounting for demographic characteristics. Like figure 1, a drop in the utilisation of ANC-4 and PNC is observed. This suggests that exposure to ASHA supports utilisation of ANC-1 and SBA.

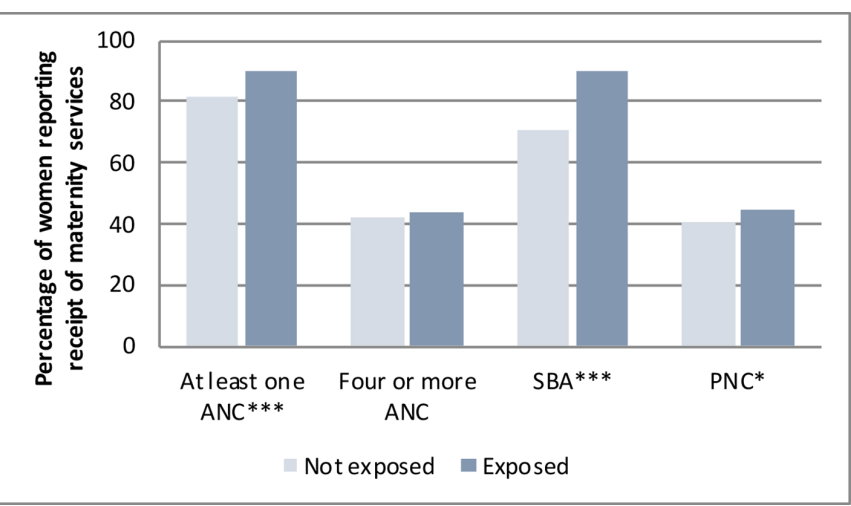

Figure 1 Cascade of maternity services received by individual Accredited Social Health Activist exposure status note: *Significant at $\mathrm{p}<0.10 ;{ }^{* \star}$ significant at $\mathrm{p}<0.05$; ${ }^{* \star *}$ significant at $\mathrm{p}<0.01$ percentages were estimated using a linear probability model to control for the following confounding variables: maternal education, maternal age, caste, religion, parity, household wealth quintile, state and rurality. ANC, antenatal care; PNC, postnatal care; SBA, skilled birth attendance. 


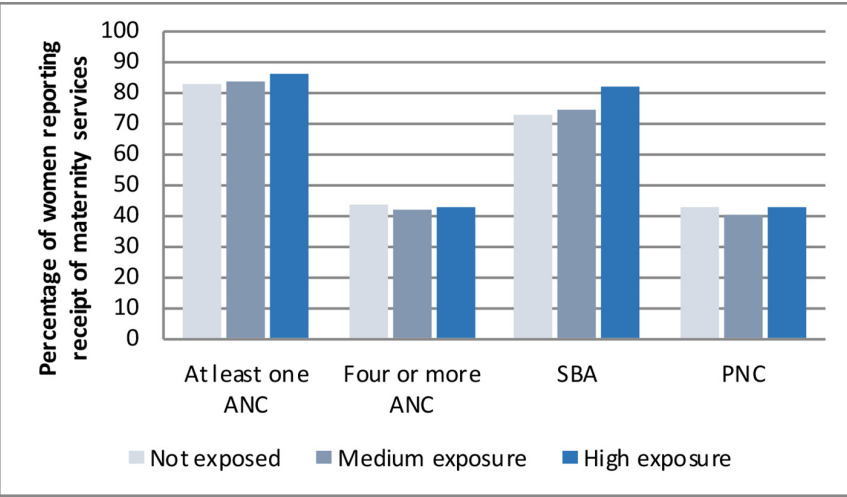

Figure 2 Cascade of maternity services by clusterlevel Accredited Social Health Activist exposure intensity adjusted for demographic variables. *Significant at $p<0.10$; **significant at $\mathrm{p}<0.05$; ${ }^{* \star *}$ significant at $\mathrm{p}<0.01$; percentages were estimated using a linear probability model to control for the following confounding variables: maternal education, maternal age, caste, religion, parity, household wealth quintile, state and rurality. ANC, antenatal care; PNC, postnatal care; SBA, skilled birth attendance.

\section{Association between exposure to ASHA and number of services} Exposure to ASHA was associated with an 8.8 percentage point (95\% CI: -0.102 to -0.074 ) reduction in receiving no maternity services, a 12 percentage point (95\% CI: 0.091 to 0.151 ) increase in receiving some services and no effect on receiving all the services (table 3 ).

Medium to high cluster-level ASHA exposure intensity is associated with a decrease in women receiving no services (two percentage points decrease for medium exposure, and six percentage points decrease for high exposure), and increase in women receiving some services (7.1 percentage points increase for medium exposure, and 12.7 percentage points increase for high exposure), compared with no ASHA exposure. However, a negative association is seen between ASHA exposure and women receiving all services. Exposure to ASHA is thus strongly associated with the service initiation and continuation along the maternity continuum (ie, receiving some services), but not associated with the completion of service utilisation along the continuum (table 4).

To further understand the effect of ASHA exposure on the number of services, we break down the 'some' category into the exact number of services. Exposure to
ASHA is associated with a $9 \%$ (95\% CI: -0.102 to -0.074$)$ reduction in women receiving no services, and a $6 \%$ (95\% CI: -0.080 to -0.039 ) reduction in women receiving only one service. A significant increase in $6 \%(95 \% \mathrm{CI}$ : $0.027-0.095)$ and $12 \%$ (95\% CI: $0.090-0.156)$ is seen in women receiving two and three services, respectively (figure $3 \mathrm{a}$ and $\mathrm{b}$ ). The marginal effect of ASHA exposure on women receiving all the four services is negligible.

\section{DISCUSSION}

CHWs such as the ASHA are in a unique position to bring women who may not typically use formal health services, into the healthcare system. Our study indicates that exposure to the ASHA is associated with an increased probability of women receiving at least one ANC and SBA. In terms of number of services, exposure to ASHA accounts for a $12 \%$ increase in women receiving at least some of the services, and a 9\%crease in women receiving no services. However, exposure to ASHA does not increase the likelihood of women using all the services along the continuum.

We further explore why the presence of an ASHA, though effective in women initiating and continuing along the maternity continuum, does not result in the completion of services along the continuum. When women have two or three services, ANC-4 and PNC are most likely to be missed out (table 2). A number of social, economic and cultural barriers, as well as cognitive and supply-side factors may limit the use of four or more ANC visits. ${ }^{24}{ }^{25}$ Women may not understand the importance of ANC, especially if they have a low-risk pregnancy. An initial ANC visit typically involves a general check-up; women may not perceive this as important and assume that their health situation will remain unchanged until the time of delivery. Women may also not have a positive experience during their first ANC visit due to a variety of service provision reasons such as long waiting times, provider attitudes and quality of services; this, in turn, will affect their willingness to return. ${ }^{24}$ Additionally, women may forget when it is time for them to go back to the provider, as in the case for other health conditions that require ongoing follow-up care. ${ }^{25}$ Furthermore, a variety of cultural and economic factors such as family support and ability to pay might dictate a woman's ability

Table 3 Association between individual exposure to ASHA and number of services utilised, using a multinomial logistic regression model†

\begin{tabular}{lllc}
\hline & \multicolumn{2}{l}{ Predicted probability $(95 \% \mathrm{Cl})$} & Marginal effects (95\% Cl) \\
\cline { 2 - 3 } & ASHA exposure & No ASHA exposure & ASHA versus no ASHA \\
\hline No services & $0.03(0.016$ to 0.034$)$ & $0.11(0.099$ to 0.127$)$ & $-0.088(-0.102 \text { to }-0.074)^{\star \star}$ \\
Some services & $0.80(0.776$ to 0.826$)$ & $0.68(0.661$ to 0.698$)$ & $0.121(0.091 \text { to }-0.151)^{\star \star}$ \\
All services & $0.17(0.149$ to 0.198$)$ & $0.21(0.190$ to 0.224$)$ & $-0.033(-0.062 \text { to }-0.005)^{\star}$ \\
\hline
\end{tabular}

*Significant at $0.05 ;{ }^{* *}$ significant at $\mathrm{p}<0.01$.

†Model controls for the following confounding variables: maternal education, maternal age, caste, religion, parity, household wealth quintile, state and rurality.

ASHA, Accredited Social Health Activist. 
Table 4 Association between cluster-level ASHA intensity and number of services utilised, using a multinomial logistic regression model†

\begin{tabular}{|c|c|c|c|}
\hline No. of services & Exposure intensity & $\begin{array}{l}\text { Predicted probability } \\
(95 \% \mathrm{Cl})\end{array}$ & Marginal effects $(95 \% \mathrm{Cl})$ \\
\hline \multirow[t]{3}{*}{ No services } & No ASHA exp. & $0.113(0.091-0.135)$ & $(\operatorname{Ref})$ \\
\hline & Some ASHA exp. & $0.090(0.073-0.105)$ & $-0.024(-0.049 \text { to }-0.002)^{\star}$ \\
\hline & High ASHA exp. & $0.053(0.041-0.066)$ & $-0.060(-0.085 \text { to }-0.034)^{\star * *}$ \\
\hline \multirow[t]{3}{*}{ Some services } & No ASHA exp. & $0.659(0.629-0.690)$ & $(\operatorname{Ref})$ \\
\hline & Some ASHA exp. & $0.730(0.709-0.751)$ & $0.071(0.032-0.109)^{\star \star \star}$ \\
\hline & High ASHA exp. & $0.786(0.756-0.817)$ & $0.127(0.082-0.172)^{\star \star \star}$ \\
\hline \multirow[t]{3}{*}{ All services } & No ASHA exp. & $0.228(0.203-0.252)$ & $(R e f)$ \\
\hline & Some ASHA exp. & $0.180(0.160-0.200)$ & $-0.047(-0.078 \text { to }-0.016)^{\star * *}$ \\
\hline & High ASHA exp. & $0.160(0.131-0.189)$ & $-0.067(-0.106 \text { to }-0.028)^{\star \star}$ \\
\hline
\end{tabular}

*Significant at $\mathrm{p}<0.10 ;{ }^{* *}$ significant at $\mathrm{p}<0.05 ;{ }^{* * *}$ significant at $\mathrm{p}<0.01$.

†Model controls for the following confounding variables: maternal education, maternal age, caste, religion, parity, household wealth quintile, state and rurality.

ASHA, Accredited Social Health Activist.

to return for ANC. Arguably, ASHA can play an important role in addressing several of these factors to encourage women, at the community level, to go for ANC visits. Training ASHA to recognise and address these barriers is important to improving the coverage of ANC-4.

$\mathrm{PNC}$ is only recently receiving focus as a key maternal and newborn health intervention and the factors affecting its uptake are not well understood. ${ }^{26}$ High levels of dropouts at the level of PNC observed in our study have also been reported by studies conducted in other countries. ${ }^{9}$ Women who deliver in a health facility are more likely to receive a postnatal check-up within 2 days of giving birth compared with women who deliver at home ${ }^{5}$; home births, especially those without any skilled attendance, place the responsibility of a postnatal check-up on the mother. In our study, over $30 \%$ of women who have a skilled attendant do not go on to receive any PNC. To understand the factors affecting this dropout, we need to closely examine who the providers of SBA and PNC are and where these services are being delivered. Some country programme are training CHWs to conduct PNC checks at home. The WHO endorses this strategy in areas where there are shortages of formal health workers. ${ }^{27}$
Another important factor to consider in interpreting results about PNC coverage is the manner in which questions about a postnatal health check-up and its timing are asked and interpreted by the women. Possibly, women and newborns with complications may be separated after birth, women may not recall having a health check-up immediately after giving birth, or may not consider it noteworthy.

We observed a dose-response relationship between the 'intensity' of ASHA exposure and the number of services utilised. Medium exposure to ASHA was associated with a 7\% increase in women utilising some of the services, compared with a $13 \%$ increase associated with high ASHA exposure. This finding may be interpreted in a few ways: (1) Increased ASHA activity (measured as 'intensity' of exposure) may result in improved service utilisation. (2) There is an indirect community effect resulting from a higher percentage of pregnant women having access to health information. Positive behaviour change among some women in the community influences other women to improve their health-seeking behaviours and (3) Greater ASHA exposure may be reported in areas where community health infrastructure is stronger, and
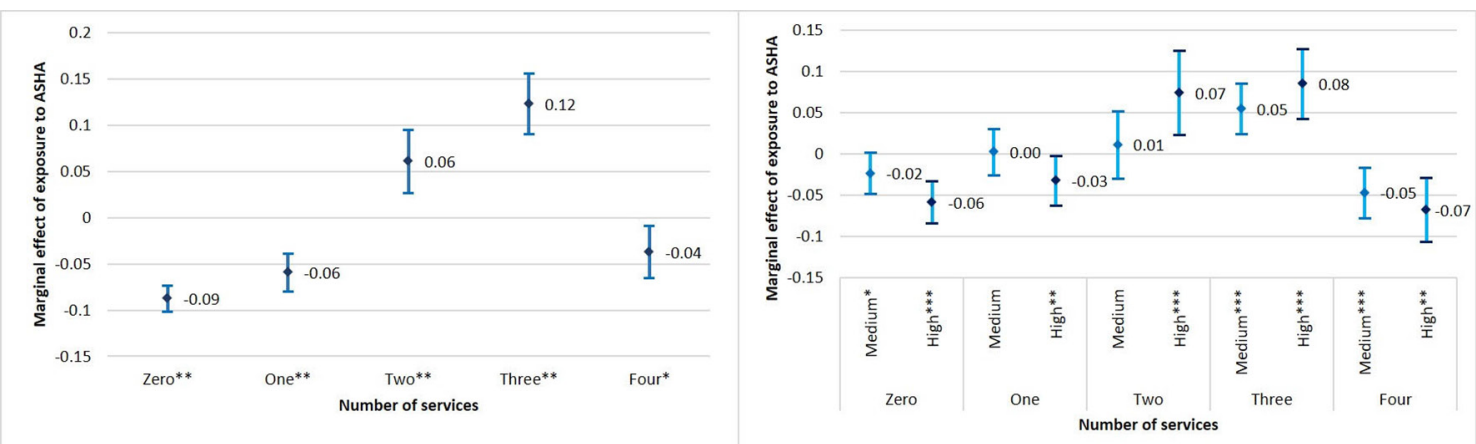

Figure 3 (A) Marginal effects of individual ASHA exposure on number of maternity services. (b) Marginal effects of clusterlevel ASHA exposure intensity on number of maternity services. ASHA, Accredited Social Health Activist. 
therefore more women are likely to use health services compared with areas that have poor health infrastructure.

This analysis is an advance over other studies in that it is based on a nationally representative sample population. The survey provides data on a range of demographic variables, which allowed us to control for potential confounders. This is the first study to assess the association between a national-level CHW programme and women's retention in the maternity continuum. However, our findings must be interpreted within the context of the study design. First, the effect of the ASHA is limited by several systemic factors. While the ASHA can influence certain health knowledge and health-seeking behaviours, she cannot account for factors such as lack of facility-based services, poor geographical and financial accessibility. Second, as a cross-sectional study, our findings are descriptive and highlight important associations that should be further investigated using more robust designs and qualitative studies. Specifically, association between exposure to the ASHA and the uptake of maternity services might be confounded by the overall strength of the health system. Areas in which the ASHA programme is not functioning well might also be areas where the health system is weak or other important government maternity subsidy programme (such as the Janani Suraksha Yojana programme) are not working as well. Third, as has been noted by other studies, measuring the coverage of services, while important, does not capture the quality of service provision. ${ }^{28-30}$ For example, global coverage of births with a skilled birth attendant has increased from $57 \%$ to $74 \%$, of at least one ANC from $65 \%$ to $83 \%$, and of four or more ANC visits from $37 \%$ to $64 \%$. However, several countries with high coverage continue to have poor maternal and child survival. ${ }^{29}$

\section{CONCLUSION}

The role of CHWs is critical, especially in the current global health ecosystem where skilled human resources for health are a glaring challenge in most low-income countries. Our study suggests that the presence of an ASHA in a community encourages positive healthcare-seeking behaviours along the continuum of maternity care. Effective strategies to strengthen such CHW programme warrant further evaluation.

Acknowledgements We thank to Catherine Zimmer for her valuable guidance on the analysis and the Odum Institute for research support. We would also like to thank the principal investigators of the IHDS surveys- Sonalde Desai and Reeve Vanneman of the University of Maryland and the National Economic Council of Applied Economic Research; and the IHDS management and survey teams.

Contributors SA and SC designed the research protocol. All authors reviewed and contributed to the design of the protocol. Analysis was conducted, and first draft of the manuscript was prepared by SA. All authors contributed substantially to reviewing the methods, interpreting and contextualising the study results. All authors reviewed, contributed to and approved the final manuscript.

Funding We are grateful to the Carolina Population Center and NIH/NICHD for training support (T32 HD007168) and for general support (P2C HD050924).

Competing interests None declared.

Patient consent for publication Not required.
Ethics approval Ethical approval was received from the University of North Carolina, Chapel Hill Institutional Review Board.

Provenance and peer review Not commissioned; externally peer reviewed.

Data availability statement The data for this study are publicly available through the National Economic Council of Applied Economic Research.

Open access This is an open access article distributed in accordance with the Creative Commons Attribution Non Commercial (CC BY-NC 4.0) license, which permits others to distribute, remix, adapt, build upon this work non-commercially, and license their derivative works on different terms, provided the original work is properly cited, appropriate credit is given, any changes made indicated, and the use is non-commercial. See: http://creativecommons.org/licenses/by-nc/4.0/.

\section{REFERENCES}

1. Kerber KJ, de Graft-Johnson JE, Bhutta ZA, et al. Continuum of care for maternal, newborn, and child health: from slogan to service delivery. The Lancet 2007;370:1358-69.

2. Abou-Zahr I, Lidia C, Wardlaw TM. Antenatal care in developing countries promises. achievements and missed opportunities. 2003.25/12/ 2014.

3. Lincetto O, Mothebesoane-anoh S, Gomez P, et al. Opportunities for Africa's Newborns: Antenatal Care 2013.

4. Stanton C, Blanc AK, Croft T, et al. Skilled care at birth in the developing world: progress to date and strategies for expanding coverage. J Biosoc Sci 2007;39:109-20.

5. Wang W, Alva S, Wang S.Levels and Trends in the Use of Maternal Health Services in Developing Countries. DHS Comparative Reports No. 26. Calverton, Maryland, USA: ICF Macro. DHS Comp Reports No 26 Calverton, Maryland, USA ICF Macro; 2011.

6. Beeckman K, Louckx F, Masuy-Stroobant G, et al. The development and application of a new tool to assess the adequacy of the content and timing of antenatal care. BMC Health Serv Res 2011;11.

7. Barros H, Tavares M, Rodrigues T. Role of prenatal care in preterm birth and low birthweight in Portugal. J Public Health 1996;18:321-8.

8. Wang W, Hong R. Levels and determinants of continuum of care for maternal and newborn health in Cambodia-evidence from a population-based survey. BMC Pregnancy Childbirth 2015;15.

9. Singh K, Story WT, Moran AC. Assessing the continuum of care pathway for maternal health in South Asia and sub-Saharan Africa. Matern Child Health J 2015.

10. Darmstadt GL, Bhutta ZA, Cousens S, et al. Evidence-based, costeffective interventions: how many newborn babies can we save? The Lancet 2005;365:977-88.

11. Kumar V, Mohanty S, Kumar A, et al. Effect of community-based behaviour change management on neonatal mortality in Shivgarh, Uttar Pradesh, India: a cluster-randomised controlled trial. The Lancet 2008;372:1151-62.

12. Yeji F, Shibanuma A, Oduro A, et al. Continuum of care in a maternal, newborn and child health program in Ghana: low completion rate and multiple obstacle factors. PLoS One 2015;10:e0142849.

13. Haines A, Sanders D, Lehmann U, et al. Achieving child survival goals: potential contribution of community health workers. The Lancet 2007;369:2121-31.

14. Bhutta ZA, Darmstadt GL, Hasan BS, et al. Community-based interventions for improving perinatal and neonatal health outcomes in developing countries: a review of the evidence. Pediatrics 2005;115(2 Suppl):519-617.

15. Perry HB, Zulliger R, Rogers MM. Community health workers in low-, middle-, and high-income countries: an overview of their history, recent evolution, and current effectiveness. Annu Rev Public Health 2014;35:399-421.

16. Bhutta ZA, Ali S, Cousens S, et al. Interventions to address maternal, newborn, and child survival: what difference can integrated primary health care strategies make? The Lancet 2008;372:972-89.

17. Perry HZR. How effective are community health workers?. 2013, 2014. Available: http://www.coregroup.org/storage/Program Learning/Community_Health_Workers/review of chw effectiveness for mdgs-sept2012.pdf

18. Perry H, Crigler L, Hodgins S, et al. Developing and strengthening community health worker programs at scale a reference guide and case studies for program managers and.

19. Bajpai N, Dholakia R. Improving the performance of accredited social health activist in India 2011.

20. Desai S, Vanneman R.India human development Survey-II (IHDS-II) 2011-12 user guide. New Delhi, India; Ann Arbor, MI, USA; 2015.

21. Department of Reproductive Health and Research. Proportion of births attended by a skilled health worker 2008 updates, 2008. 
Available: http://apps.who.int/iris/bitstream/10665/69950/1/WHO_ RHR_08.22_eng.pdf [Accessed 15 Dec 2016].

22. Williams R. Using the margins command to estimate and interpret adjusted predictions and marginal effects. The Stata Journal 2012;12:308-31.

23. Kolenikov S, Angeles G. Socioeconomic status measurement with discrete proxy variables: is principal component analysis a reliable answer? Rev Income Wealth 2009;55:128-65.

24. Lincetto O, Mothebesoane-Anoh S, Gomez P, et al. Antenatal care. In: Lawn J, Kerber K, Save the Children, eds. Opportunities for Africa's Newborns. World Health Organization, 2006: 51-62.

25. Martin LR, Williams SL, Haskard KB, et al. The challenge of patient adherence. Ther Clin Risk Manag 2005;1:189-99.
26. Warren C, Daly P, Toure L, et al. Postnatal care. Oppor Africa's Newborns:1-12.

27. World Health Organization,, UNICEF. Home visits for the newborn child:a strategy to improve survival. Switzerland Geneva; 2009.

28. Miller S, Abalos E, Chamillard M, et al. Beyond too little, too late and too much, too soon: a pathway towards evidence-based, respectful maternity care worldwide. The Lancet 2016;388:2176-92.

29. Campbell OMR, Calvert C, Testa A, et al. The scale, scope, coverage, and capability of childbirth care. The Lancet 2016;388:2193-208.

30. Koblinsky M, Moyer CA, Calvert C, et al. Quality maternity care for every woman, everywhere: a call to action. The Lancet 2016;388:2307-20. 